Revue des patrimoines

25 | 2014

Le patrimoine de la Grande Guerre

\title{
La conscience du monument en Suisse. De l'appropriation à la mnémotechnologie
}

\section{Nicole Bauermeister}

\section{(2) OpenEdition \\ 1 Journals}

Electronic version

URL: http://journals.openedition.org/insitu/11509

DOI: 10.4000/insitu.11509

ISSN: 1630-7305

\section{Publisher}

Ministère de la Culture

\section{Electronic reference}

Nicole Bauermeister, « La conscience du monument en Suisse. De l'appropriation à la

mnémotechnologie », In Situ [Online], 25 | 2014, Online since 12 December 2014, connection on 26

June 2020. URL : http://journals.openedition.org/insitu/11509 ; DOI : https://doi.org/10.4000/insitu. 11509

This text was automatically generated on 26 June 2020 .

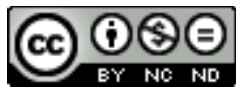

In Situ Revues des patrimoines est mis à disposition selon les termes de la licence Creative Commons Attribution - Pas d'Utilisation Commerciale - Pas de Modification 4.0 International. 


\title{
La conscience du monument en Suisse. De l'appropriation à la mnémotechnologie
}

\author{
Nicole Bauermeister
}

\section{La Société d'histoire de l'art en Suisse en quelques mots}

1 Dans le cadre de ce festival qui a pour thème la Suisse, ainsi que la collection, la tentation serait grande de vous parler de l'esthétique des collections, de leur philosophie ou de leur histoire. La Suisse reste un pays relativement neuf en matière de grandes collections, puisqu'il n'y a pas eu de grandes collections royales ou aristocratiques à proprement parler.

2 Il y a des gens plus compétents que moi dans ce domaine, qui ont étudié tout cela sous différents aspects et qui vont en parleront autre part dans le cadre de ce festival.

3 Je vais quant à moi vous parler d'un défi autrement plus important - un défi politique et de la relation de la population suisse avec la plus grande collection de son pays.

4 En France, on est très familier avec le concept d'exception culturelle. C'est quelque chose que l'on connaît bien aussi en Suisse. En effet, la Suisse est composée de 26 cantons et demi-cantons, et s'accommode quotidiennement de 26 exceptions culturelles, que ce soit sur le plan de la culture, de la langue ou de la religion (comme on le sait, la Suisse est quadrilingue et a deux principales confessions). La notion même de «culture suisse » est donc déjà un défi majeur ; mais cette culture est une réalité, parce que l'initiative de personnalités privées a contribué à la faire naitre et exister.

5 L'architecture en tant qu'art « utile » en trois dimensions, diverse du point de vue des décors et des styles mais également des fonctions et des affectations, constitue sans doute la meilleure illustration, le meilleur symbole, de l'identité pluriculturelle de la Suisse. 
6 La Société d'histoire de l'art en Suisse, dont j'assure la direction, est une société privée, fondée il y a 134 ans, qui a contribué de manière déterminante à ce que la population suisse puisse s'« approprier » la plus grande collection collective et publique du pays. L'impulsion qui a présidé à la création de la Société d'histoire de l'art en Suisse est due à des privés - historiens de l'art ou grands bourgeois qui ont créé une association dans le but délibéré de permettre à la population de connaître et de s'approprier la plus grande collection de son pays - je veux parler ici de l'ensemble du patrimoine culturel et bâti de Suisse (fig. 1).

\section{Figure 1}

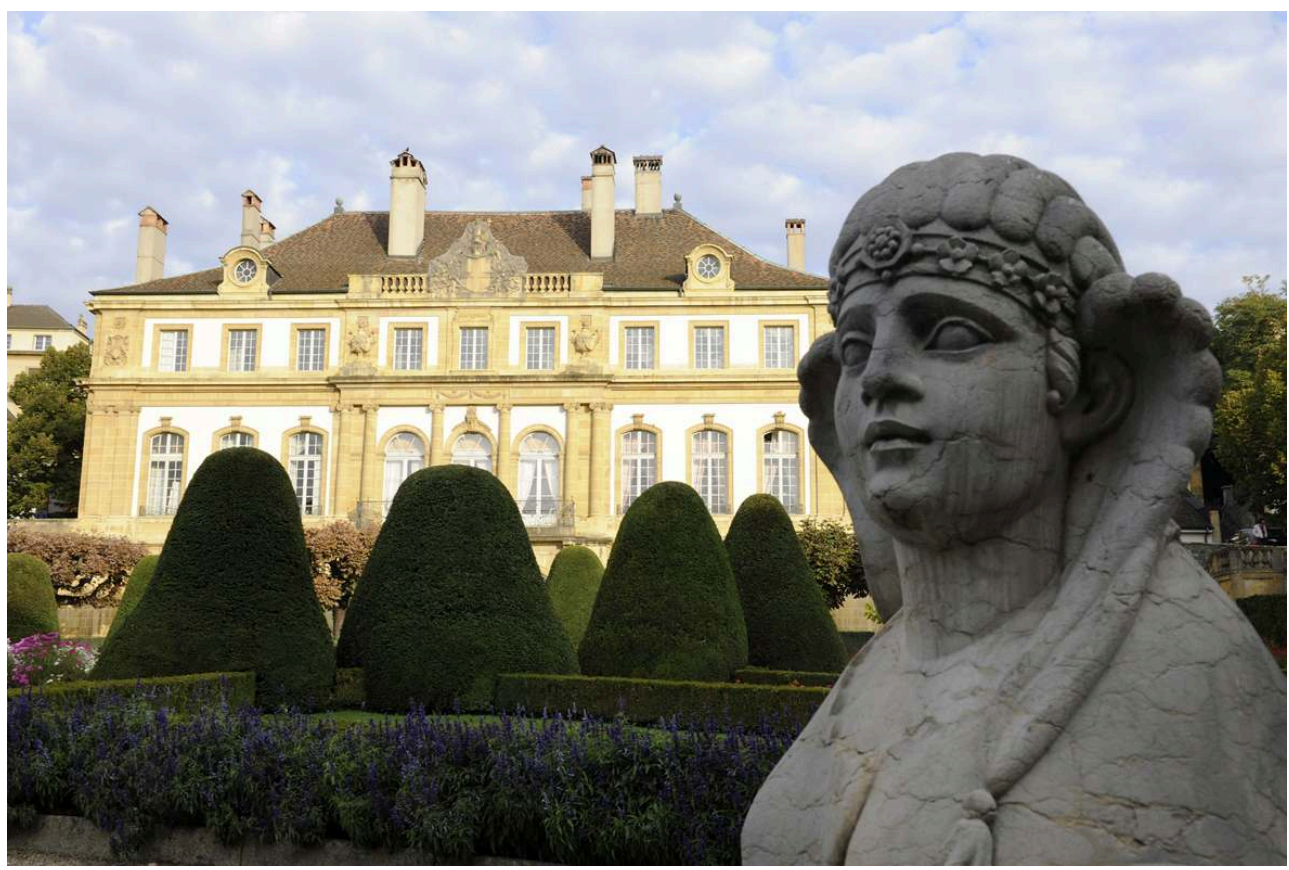

Neuchâtel (Suisse), Hôtel Du Peyrou.

Phot. Sterchi, Renaud. (C) Société d'histoire de l'art en Suisse, 2017.

Qu'est-ce que la Société d'histoire de l'art en Suisse, comment fonctionne-t-elle? Pourquoi ses buts patrimoniaux ont-ils été déclarés d'utilité publique? Comment contribue-t-elle, grâce à ce qu'elle a mis en œuvre depuis 1880, à la cohésion culturelle du pays?

La Société d'histoire de l'art en Suisse SHAS, ou GSK pour évoquer l'acronyme allemand de la Geselleschaft für schweizerische Kunstgeschichte, est la plus ancienne et la plus importante société d'utilité publique active dans le domaine culturel de Suisse. Elle est importante par le nombre de sesmembres mais aussi par celui de ses réalisations.

Elle occupe une position unique dans le paysage culturel suisse. Cette position est due à son histoire; ses liens avec la Confédération (créée en 1848) et les cantons sont étroits en raison des travaux d'inventaire menés depuis 1920 pour les « Monuments d'art et d'histoire de la Suisse ». Les « Monuments d'art et d'histoire de la Suisse » sont de facto un inventaire national, mais cet inventaire est géré par une société privée dont les tâches se sont substituées à celles de l'État. Le financement de l'inventaire est assuré par les membres de la SHAS, les pouvoirs publics, et de plus en plus par des fonds privés par le biais du mécénat. 
10 La SHAS s'appuie sur une longue tradition, mais est une société très innovante. Elle publie et gère des ouvrages scientifiques, des ouvrages destinés à un large public, des publications imprimées et numériques ayant pour buts l'éducation et la formation, destinées aux particuliers comme aux institutions.

\section{Les origines de la "Société d'histoire de l'art en Suisse ", ou le moment où l'identité de la Suisse en tant que nation se construit grâce à la prise de conscience du patrimoine culturel}

11 Nous sommes en 1880 , soit trente-deux ans après la fondation de l'État fédéral, en pleine révolution industrielle. Des fortifications sont détruites, des tunnels comme celui du Gothard percés, des lignes de chemin de fer tracées, de nombreux ports de plaisance construits. Le tourisme de la grande bourgeoisie se développe ; de nombreux bâtiments et monuments de l'Ancien Régime sont alors détruits, sacrifiés non au progrès, mais à l'idée que l'on s'en fait alors.

12 Cette même année, en 1880, la "Société patriotique pour la conservation des monuments historiques » est fondée. Elle ne deviendra la « Société d'histoire de l'art en Suisse » que cinquante-quatre ans plus tard, en 1934. Sa création émane, comme nous l'avons laissé entendre, de personnalités membres de la "Société suisse des beauxarts $»^{1}$; son premier président est le peintre genevois Théodore de Saussure, petit-fils du naturaliste Horace Bénédict de Saussure et cousin du linguiste Ferdinand de Saussure.

13 La tâche de la Société consiste alors à " attirer l'attention du public sur les monuments historiques et les trésors artistiques de la Suisse » et à «contribuer directement ou indirectement à leur conservation ».

14 La crainte que des édifices ou des objets d'art importants ne soient détruits ou vendus à l'étranger fit rapidement sentir la nécessité d'en établir un inventaire.

15 En 1915, la SHAS se mit en rapport avec le Département fédéral de l'Intérieur (par département, il faut entendre ministère), la Commission fédérale des Monuments historiques, le Musée national suisse et la Société suisse de préhistoire pour examiner les possibilités d'établir un inventaire national des biens culturels du pays. Cet inventaire se fondait sur les méthodes de la Statistique des monuments d'art et d'histoire en Suisse établie en 1872 par Johann Rudolf Rahn (1841-1912) ${ }^{2}$. Le premier volume de la série, rédigé par Linus Birchler, parut en 1927. Il était consacré à trois districts du canton de Schwyz, à savoir Einsiedeln, March et Höfe.

16 C'est ainsi que naquit le «fer de lance " de la SHAS : la collection des «Monuments d'art et d'histoire de la Suisse ».

\section{Les Monuments d'art et d'histoire de la Suisse, période de stabilité et d'appropriation psychologique du patrimoine culturel}

En 1925, d'entente avec le Département fédéral de l'Intérieur et avec diverses sociétés scientifiques, la SHAS entreprit donc l'inventaire national des Monuments d'art et d'histoire de la Suisse, en étroite collaboration avec les cantons ; elle assura également sa publication. Le premier volume parut en 1927. 
La série se fonde sur une démarche topographique et est subdivisé en séries cantonales. Chaque volume recense le patrimoine architectural et artistique d'une région donnée, entre la fin de l'Antiquité et le début de l'époque moderne; il lui consacre une analyse détaillée, permettant une comparaison sur le plan national. Le texte de l'inventaire tient compte des données historiques, artistiques et culturelles de chaque époque.

La méthode et les critères de sélection appliqués se basent, dans un premier temps, en grande partie sur les inventaires réalisés en France, dont celui créé en juillet 1834 à l'initiative de François Guizot, alors ministre de l'Instruction publique ${ }^{3}$, inventaires qui allaient être suivis dès le milieu du siècle par les inventaires des monuments historiques en Allemagne, Autriche et Angleterre.

Toutefois, la SHAS, sur le modèle de la Statistique de J.R. Rahn mais surtout sur celui de l'œuvre de Durrer, opte rapidement pour un traitement à égalité des différents styles anciens, refusant la hiérarchisation des périodes. Selon les responsables de l'institution, un objet devait être placé dans son contexte historique et jugé en fonction de sa valeur, en comparaison avec les autres créations d'une même époque. Une grande importance était accordée à la conservation aussi complète que possible de l'œuvre authentique et à la distinction de ce qui, dans un monument restauré, était d'origine par rapport à ce qui était reconstitué, de manière à éviter tout pastiche.

21 Les auteurs des premiers volumes des « Monuments d'art et d'histoire » considéraient avant tout leurs ouvrages comme une histoire régionale à l'attention de la population locale, et comme un recueil de sources pour de futures recherches. L'exigence scientifique devint nettement plus marquée à partir des années 1940.

2 Du point de vue administratif, comme nous l'avons laissé entendre, ce projet est porté par un partenariat public-privé entre la Société d'histoire de l'art en Suisse et les Cantons, et soutenu subsidiairement par la Confédération. La SHAS pilote l'ensemble du projet, garantit sa qualité scientifique et publie les résultats des recherches; les travaux d'inventaire et de recherche à proprement parler s'effectuent de manière décentralisée, dans les cantons mêmes, et sont financés par ces derniers.

Actuellement, quelque 40 historiens de l'art répartis dans 17 cantons sont en train de préparer 22 volumes. Ce sont en règle générale des employés des Cantons, qui travaillent en étroite collaboration avec les Services des monuments historiques cantonaux.

Plus de 230 institutions dans 20 pays sont abonnées aux Monuments d'art et d'histoire de la Suisse - dont 127 institutions scientifiques et étatiques de Suisse ${ }^{4}$.

\section{Développement numérique}

Depuis 2012, l'édition papier de chaque nouveau volume se double d'une édition numérique. Cette dernière, dont le texte est identique à celui de l'édition papier présente de nombreuses plus-values : tous les avantages du livre numérique (absence d'encombrement, lecture différée, taille et types des caractères ajustables, annotations, recherche plein texte, table des matières dynamique), mais surtout géolocalisation des éléments répertoriés, possibilité de voir concrètement l'édifice dans son contexte grâce aux vues satellites, meilleure compréhension d'ensemble du bâtiment grâce aux vues de type street view (fig. 2). 
Figure 2
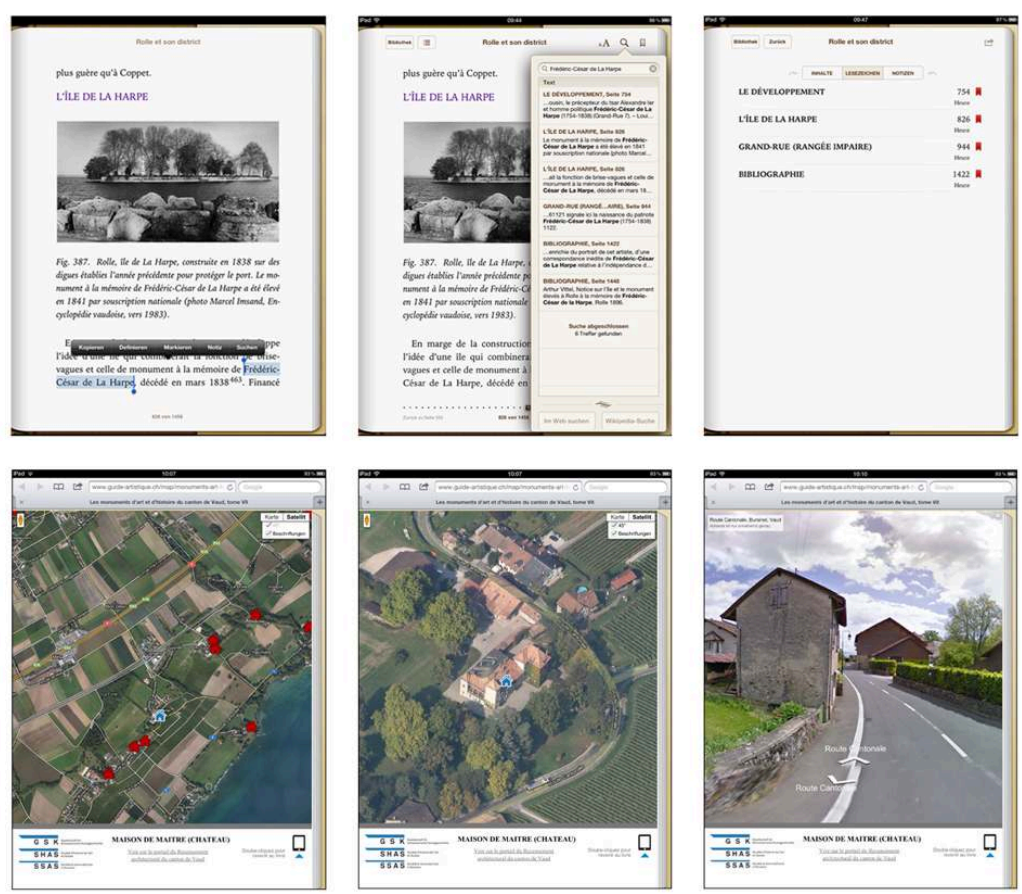

Quelques aspects d'une publication des « Monuments d'art et d'histoire de la Suisse » en version numérique.

(c) Société d'histoire de l'art en Suisse, 2013.

Last but not least, la version eBook des MAH offre un lien direct avec les sites internet des instances en charge des bâtiments, donne accès aux plans d'affectation, aux valeurs de recensement, aux informations directes sur les zones à protéger et aux notes de classement des bâtiments. Un lien avec les grands dictionnaires spécialisés tels le SIKART de l'Institut d'histoire de l'art de Zürich, est en outre proposé.Il s'agit donc d'un outil de travail unifié particulièrement utile aussi pour toutes les professions des domaines de l'art, de l'architecture, de l'aménagement du territoire et de l'urbanisme ${ }^{5}$.

La version numérique des Monuments d'art et d'histoire est ce que l'on peut qualifier de courroie de transmission entre les historiens de l'art et les décideurs, puisqu'elle met entre les mains de ces derniers le résultat des recherches scientifiques, également accessibles au public le plus large.

À terme, la SHAS a pour projet de rétronumériser la totalité de la collection et de la transformer en une Encyclopédie libre du patrimoine suisse - avec un accès totalement gratuit.

Pour résumer cet historique, les « Monuments d'art et d'histoire » ont avant tout été pensés à leur début comme un inventaire destiné à la population locale; il s'agissait alors d'une forme d'attestation d'identité et d'histoire commune. Dans les années d'après-guerre, ils ont davantage été conçus comme un outil scientifique. Aujourd'hui, notamment grâce à l'introduction des outils mnémotechnologiques, ils sont devenus un outil de communication et un instrument décisionnel dans le domaine du patrimoine culturel de la Suisse. 


\section{Les autres projets de la Société d'histoire de l'art en Suisse, où l'on assiste au passage progressif de la conscience personnelle du monument à une vision pédagogique et sociale de l'environnement culturel}

Depuis les années 2000, on assiste, au niveau de la conscience politique et de la gestion de la culture, à un passage progressif du concept de bâtiment, ou de monument, considéré dans son individualité à celui d'environnement culturel global, mouvement déjà amorcé au cours des années d'après-guerre.

31 Mais on constate aussi que le public change : le livre «classique » est plutôt destiné à un interlocuteur " unique ", issu de la bourgeoisie cultivée. Et dans les années d'aprèsguerre, la collection était pour ainsi dire une forme de monument personnel que le lecteur construisait chez lui.

Le temps passe, donc, et les conditions de vie des générations du baby-boom changent : appartements plus petits, pratiques culturelles nettement moins marquées par l'acquisition ou l'appropriation des objets, fussent-ils des livres. On voyage davantage, on apprend plus de choses, mais d'une manière plus diffuse, plus empirique.

La culture est devenue un objet de consommation parmi d'autre; pour rester concurrentielle sur le marché, elle doit être à la disposition du consommateur sans jamais l'encombrer. Les travaux d'édition culturelle ou scientifique prennent donc des visages de plus en plus proches de ceux de la médiation culturelle, voire de la pure communication.

Les autres collections publiées par la Société d'histoire de l'art en Suisse sont nombreuses, et obéissent systématiquement aux mêmes critères de qualité scientifique. La stratégie de recherche et de communication peut s'envisager sous la forme d'une succession de cercles concentriques; tous les produits s'appuient sur le noyau scientifique qui fait la renommée de la GSK mais sont destinés à un public de plus en plus large et composite (fig. 3). La part des supports visuels, de l'image en mouvement, voire du film, est de plus en plus notable. Nous pouvons notamment citer les quelques publications suivantes. 
Figure 3

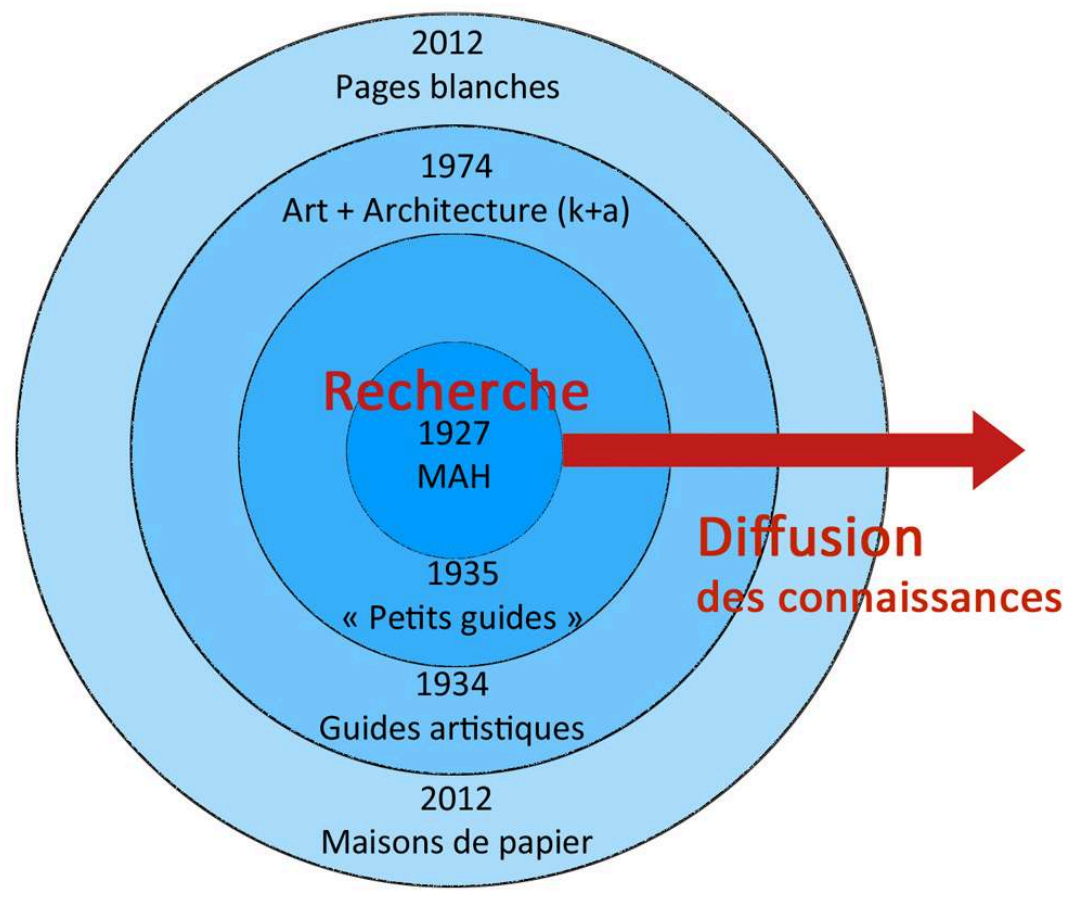

Dispositif de communication imprimée de la Société d'histoire de l'art en Suisse.

(c) Société d'histoire de l'art en Suisse, 2013.

\section{Guides d'art et d'histoire de la Suisse}

Les Guides d'art et d'histoire de la Suisse mettent en lumière la variété du patrimoine bâti de Suisse. Près de 950 numéros présentent des monuments importants comme des objets moins connus. Ce sont plus des brochures que des livres, aussi destinées à être vendues sur place, dans les monuments, légères, à coûts réduits. Créés en 1934 et souvent simplement qualifiés de "petits guides ", ils suivent le début du tourisme lié aux premiers congés payés.

Leur rythme de parution oscille entre 16 et 20 par année. Plus de 4 millions d'exemplaires ont été vendus à ce jour, dont plus de 100000 exemplaires pour certains guides (rappelons que la Suisse est un pays comportant environ 8 millions d'habitants ce qui donne la mesure de ces ventes) ${ }^{6}$.

\section{Revue Art + Architecture en Suisse}

"Art+ Architecture en Suisse ", comme son nom l'indique, est destinée à tous ceux qui s'intéressent à l'architecture, à l'histoire de l'art et à la culture en général. Cette revue concernant le patrimoine artistique et architectural de Suisse est la plus lue du pays dans son domaine. Chaque numéro est consacré à un thème spécifique, comme, par exemple, l'architecture et les décors des loges maçonniques de Suisse (fig. 4). 
Figure 4

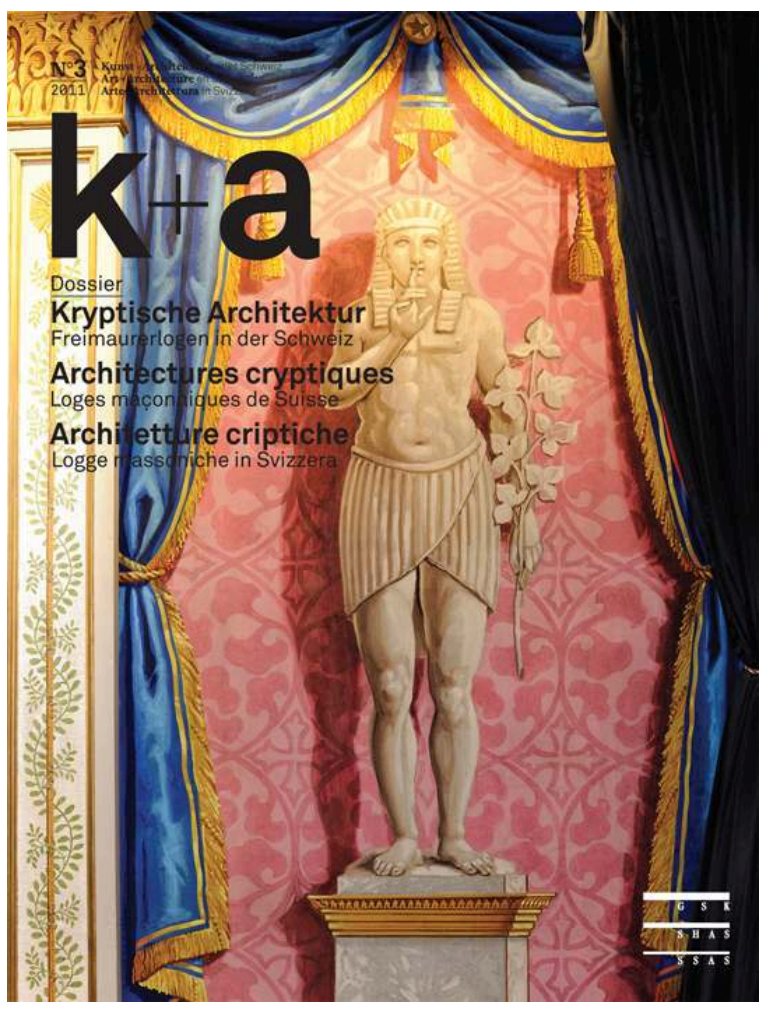

Un exemple de couverture de la revue art + architecture en Suisse consacrée à l'architecture et aux décors des temples maçonniques de Suisse.

(c) Société d'histoire de l'art en Suisse, 2011.

Elle est distribuée dans 35 pays étrangers par le biais des ambassades de Suisse.

\section{Maisons de papier}

Chacun sait que si une personne n'est pas entrée dans un musée avant 18 ans, elle a $80 \%$ de chances de moins qu'une autre d'entrer dans un musée durant le reste de sa vie. Ce fait est démontré par les statistiques de l'ICOM. Les ouvrages pour enfants de la SHAS répondent à ce besoin de formation précoce des jeunes, à cette nécessité de leur faire prendre conscience de leur environnement culturel. Ce sont des livres d'artistes, composés de deux éléments: la ville, en silhouettes découpées, dans laquelle les bâtiments importants sont représentés, et un livret à colorier. Un personnage-guide, le chat Theo, y raconte l'histoire de la ville, de ses bâtiments, de ses habitants.

\section{Pages blanches}

La série «Pages blanches » regroupe des ouvrages bilingues consacrés au patrimoine bâti ainsi qu'aux arts décoratifs, en Suisse ou en rapport avec la Suisse. La norme d'unité est thématique, les thèmes sélectionnés significatifs sur le plan national ou international. Très richement illustrée, la collection est destinée aux personnes passionnées par la culture artistique et patrimoniale sous toutes ses formes, mais pas nécessairement spécialistes du domaine. 


\section{Un exemple concret de l'usage des inventaires scientifiques: Ambassades et représentations suisses à l'étranger}

Il serait facile d'estimer que les inventaires imprimés sont avant tout des outils destinés aux musées, dépourvus d'utilité dans la vie quotidienne des citoyens. Nos diverses collections font toutefois la preuve du contraire. L'ouvrage Ambassades et représentations suisses à l'étranger fait partie de la collection "Pages blanches ", et est particulièrement représentatif de l'usage pratique d'un inventaire scientifique. Ce produit est une nouvelle fois un partenariat public-privé : sans la Confédération, la SHAS ne pouvait pas travailler et produire cet ouvrage. Mais sans la SHAS. la Confédération n'aurait eu ni l'idée ni la volonté de le produire. Or, une fois publié, le livre devient un outil de communication idéal pour la politique culturelle de la Suisse.

L'architecture est en effet l'un de reflets privilégiés de la culture d'un pays l'expression matérielle d'un état d'esprit, d'un art de vivre. Les questions posées par une architecture suisse de l'étranger - et donc précisément destinée à matérialiser l'idée que la Suisse désire que l'on se fasse d'elle - sont en tout état de cause uniques, et passionnantes.

Mais le développement d'une société d'utilité publique passe également, et peut-être surtout à l'heure actuelle, par le numérique. L'offre de la SHAS dans ce domaine s'est donc considérablement étoffée selon le même principe de cercles concentriques : cœur scientifique, communication large public (fig. 5).

Figure 5

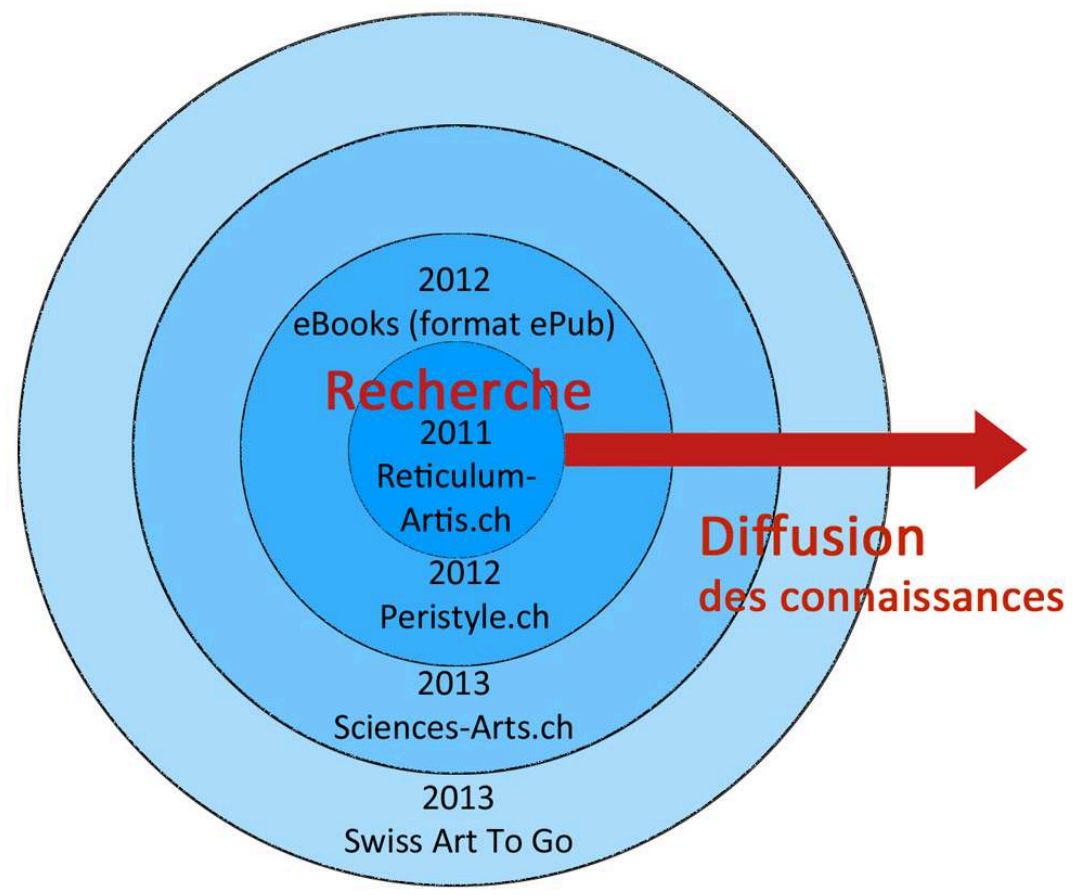

Dispositif de communication numérique de la Société d'histoire de l'art en Suisse.

(c) Société d'histoire de l'art en Suisse, 2013. 


\section{La mnémotechnologie, ou le point où des produits novateurs viennent se mettre au service de la culture afin de la rendre accessible à chacun}

Nous entrons de plein pied dans la présentation d'outils mnémotechnologiques - à savoir les outils dématérialisés permettant à la fois de fixer la mémoire culturelle et de diffuser très largement ce savoir.

L'environnement culturel a pris une autre échelle, et suit désormais une autre logique : quand les déplacements n'étaient pas particulièrement aisés, la logique régionale et topographique avait tout son sens. À l'heure de la mondialisation, cette logique est nettement moins impérative. La culture n'est plus une obligation sociale, les collections de beaux livres ne figurent plus dans les appartements comme une sorte de certificat de bonne conduite. La culture est devenue un choix - un choix de consommation parmi d'autres. Les voies d'accès à la culture se déclinent désormais de manière thématique, et plutôt selon une logique de "mass cutomization » (c'est-à-dire «si je veux, où je veux, quand je veux »).

\section{Péristyle}

6 Le référentiel Péristyle est à la fois une bibliothèque thématique en accès libre et un outil de publication en ligne ${ }^{7}$.

L'outil de publication permet de simplifier la mise en ligne des articles et publications académiques, en partant d'un document Word comme source. Chaque texte est automatiquement exporté et conservé dans différents formats (HTML, PDF, ePub). Outre la base de données trilingue et l'interface d'édition en flux tendu, Péristyle offre la possibilité de générer et d'imprimer à la demande des revues personnalisées à des coûts très concurrentiels. Le tirage est entièrement adaptable (entre 1 et 500 exemplaires).

Un tel projet est très novateur dans le domaine des sciences humaines en Suisse. Nous avons du reste de nombreux utilisateurs muets français, institutions comme particuliers (par utilisateur «muet », j'entends une personne qui se sert du système mais ne rend pas ses textes publics).

\section{Swiss Art To Go}

Swiss Art To Go fait partie des projets les plus représentatifs de la nouvelle orientation « plus large public » de notre Société (fig. 6). 
Figure 6

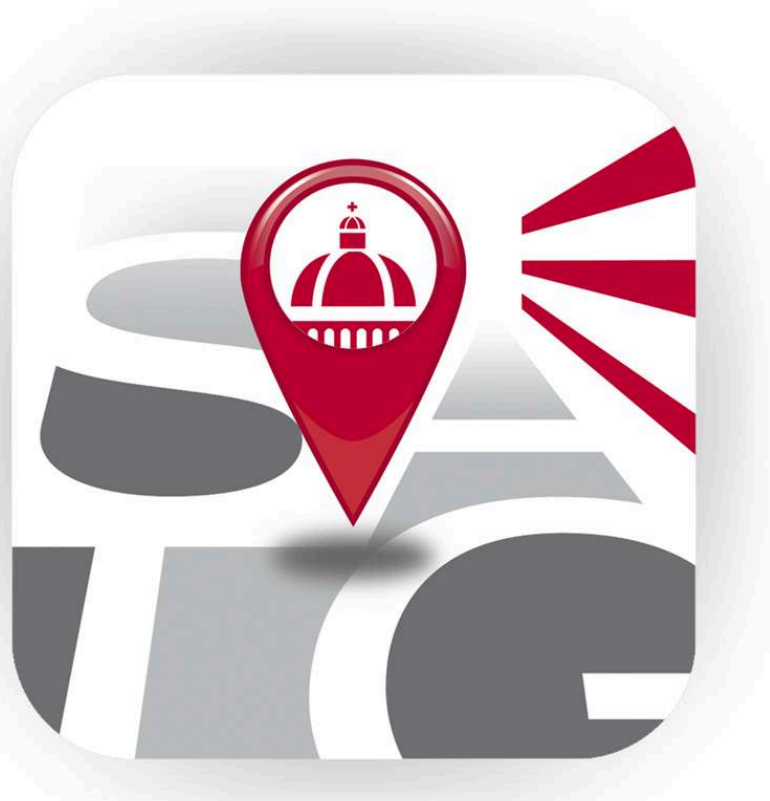

L'ICÔNE DE L'APPLICATION SWISS ART TO GO.

(c) Société d'histoire de l'art en Suisse, 2013. du plus grand audioguide du pays. Elle est destinée aux périphériques mobiles, et donne un accès rapide et aisé à l'environnement architectural et culturel. Elle est basée sur les informations issues des 4500 pages du Guide artistique de la Suisse parus depuis $1934^{8}$.

51 SATG fonctionne sur tous les périphériques mobiles usuels (Apple iOS, Androïd, Windows mobile), et est déjà très populaire en Suisse.

Grâce à la localisation GPS, SATG liste tous les éléments intéressants se trouvant soit à proximité de l'utilisateur, soit en tout endroit sélectionné. Pour chaque point d'intérêt, des informations synthétiques sont disponibles via un module de réalité augmentée. Ces informations sont accessibles sous forme de textes ou d'audioguide.

Une recherche multi-critères sophistiquée, mais d'un emploi très simple, permet en outre de faire des recherches thématiques croisées dans la riche base de données associée à l'application. Cette base regroupe des renseignements scientifiques sur pas moins de 43000 bâtiments et monuments, historiques ou contemporains (fig. 7). 
Figure 7

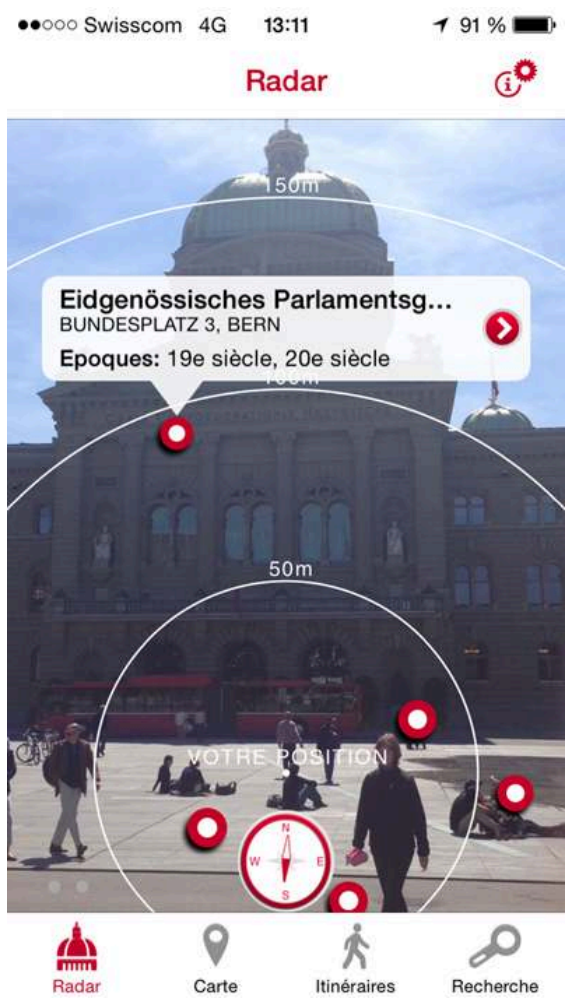

La fonction Radar de l'application Swiss Art To Go activée devant le Palais fédéral, à Berne.

(c) Société d'histoire de l'art en Suisse, 2014.

Des parcours personnalisés peuvent en outre être constitués sur la base des intérêts personnels et du temps à la disposition de l'utilisateur, comme par exemple les hôtels particuliers du XVIII siècle à Genève.

\section{Conclusion}

Les inventaires, à l'instar des autres projets de la SHAS, se distinguent par leur caractère de service aux particuliers et aux institutions. La Société d'histoire de l'art en Suisse est une société privée déclarée d'utilité publique, mais elle assure dans les faits des tâches institutionnelles types. Si ses services ont pris des visages différents au cours du temps, leurs buts n'ont en revanche pas varié

- En raison de l'évolution des technologies, la SHAS est passée de la production unique d'inventaires scientifiques imprimés à une production diversifiée largement soutenue par les outils mnémotechnologiques

- En raison de l'évolution des publics-cibles, la SHAS a tout d'abord dialogué avec les professions de l'histoire de l'art et la bourgeoisie cultivée pour s'adresser ensuite à un très large public composite. La SHAS a contribué dans un premier temps à une prise de conscience de l'identité culturelle nationale pour devenir un moteur d'intégration culturelle dans une société éminemment multiculturelle.

Pour conclure, il convient de souligner que l'apport de la SHAS dans le débat actuel lié aux énergies renouvelables s'avère tout aussi essentiel que l'étaient ses réflexions au moment de la révolution industrielle de la seconde moitié du XIX ${ }^{e}$ siècle. 
57 Nous sommes en effet dans une situation assez comparable à celle des années 1880, et la nécessaire transition énergétique génère des questions fondamentales sur la valeur des témoignages du passé face à un avenir qui n'est pas forcément et systématiquement synonyme de progrès.

58 La notion d'utilité publique dans le domaine culturel a connu différents visages depuis 135 ans, mais pas de mutation de contenu. Le but de la Société d'histoire de l'art en Suisse peut être considéré sous la forme d'un concept très à la mode aujourd'hui, celui d'interface. La technologie numérique actuelle permet précisément de favoriser l'émergence de cette interface.

59 Et cette interface donne accès de la façon la plus unifiée et la plus simple possible à une formidable diversité, celle qui constitue la Suisse. Il ne s'agit donc pas de standardiser, il s'agit au contraire de simplifier au maximum l'accès à la diversité.

\section{NOTES}

1. - Fondée en 1806 à Zofingue sous le nom de Schweizerische Geselleschaft für Künstler und Kunstfreuden - Société des artistes et des amis des arts - puis relancée après diverses turbulences politiques en 1839 sous son nom actuel; sa tâche principale était l'organisation d'expositions nationales itinérantes.

2. - La Statistique des monuments d'art et d'histoire en Suisse, publiée dès 1872 dans l'Indicateur d'Antiquités suisses (Anzeiger für Schweizerische Altertumskunde). Dans un premier temps, les principaux monuments de l'époque romane y furent répertoriés dans des listes avant d'être étendus au cours des années 1880, aux bâtiments gothiques. La méthode et les critères de sélection appliqués se basaient sur les premiers inventaires réalisés en France dès le début du $19^{\mathrm{e}}$ siècle et qui allaient être suivis dès le milieu du siècle des inventaires des monuments historiques en Allemagne, Autriche et Angleterre. La statistique atteignit son point culminant dans l'ouvrage de Robert Durrer, lequel documenta le canton d'Unterwald en plusieurs livraisons publiées de 1899 à 1928. L'ouvrage complet, qui comprend 1168 pages, se distingue nettement des inventaires réalisés à la même époque en Suisse et à l'étranger: il inclut par exemple des bâtiments réalisés jusqu'au milieu du $19^{\mathrm{e}}$ siècle ; les maisons paysannes et autres exemples de la culture rurale y sont étudiés selon les mêmes méthodes scientifiques que les édifices religieux et les constructions d'une culture plus élitiste; l'accent est mis sur la présentation de bâtiments dans leur contexte socio-culturel; toutes les sources disponibles étaient intégrées à la présentation.

3. - Le Comité pour la publication des documents inédits de l'histoire de France (Comité des travaux historiques et scientifiques) avait concrétisé la volonté du gouvernement de recenser et de protéger les monuments illustrant l'histoire nationale. Une instance spécifiquement dédiée à la connaissance, le Comité des arts et monuments, sera ainsi présidée à partir de 1837 par l'académicien et philosophe Victor Cousin. Toutefois, la logique de monument historique, avec tous les enjeux induits par sa conservation et sa restauration, devient prioritaire dans la politique de l'État par rapport à l'idée même du recensement, qui sera assumé davantage par le réseau des sociétés savantes. Il faudra attendre la fin du siècle pour voir la publication de L'Inventaire 
général des Richesses d'art de la France, sous l'égide du ministère de l'Instruction publique et des Beaux-Arts, (21 volumes entre 1878 et 1913),

4. - Voir le site : http://www.gsk.ch.

5. - Voir le site : les-monuments-d'art-et-d'histoire-du-canton-de-vaud.

6. - Voir le site : http://www.gsk.ch/fr/guides.

7. - Voir le site : www.peristyle.ch.

8. - Voir la présentation de l'application sous www.satg.ch.

\section{ABSTRACTS}

The Swiss Art History Society is a private association of public interest that carries out standard institutional mandates. It can boast of a longstanding tradition while being extremely innovative. It has made countless contributions to preserving and promoting the Swiss cultural and architectural heritage: scientific catalogues and inventories, publications targeting a wide audience, software tools and memory aids aimed at facilitating education and training, both individual and institutional, all for specific kinds of cultural consumption. Thanks in particular to the introduction of memory aids, publications once considered purely scientific have become tools for communication and cultural diplomacy, as well as important decision-making instruments in helping protect the Swiss cultural heritage.

La Société d'histoire de l'art en Suisse est une société privée d'utilité publique assurant des tâches institutionnelles types; elle s'appuie sur une longue tradition tout en étant très innovante. Ses prestations en faveur de la sauvegarde et de la mise en valeur du patrimoine culturel et bâti sont multiples: inventaires scientifiques, ouvrages large public, outils dématérialisés et mnémotechnologiques ayant pour buts l'éducation et la formation, destinés aux particuliers comme aux institutions, et répondant chacun à des usages de consommation culturelle spécifiques. Grâce, notamment, à l'introduction des outils mnémotechnologiques, les publications conçues naguère comme purement scientifiques sont devenues des outils de communication et de diplomatie culturelle, ainsi que des instruments décisionnels majeurs dans le domaine du patrimoine culturel suisse.

\section{INDEX}

Mots-clés: Inventaire scientifique, patrimoine bâti, publications, édition, transmission des savoirs, mnémotechnologie, diffusion des connaissances, utilité publique

\section{AUTHOR}

NICOLE BAUERMEISTER

directrice de Société d'histoire de l'art en Suisse bauermeister@gsk.ch 